\title{
Mycobacterium tuberculosis Presenting as Septic Shock with ARDS: Multiculturalism Promotes Early Therapy
}

\section{McGee $\mathbf{W T}^{1^{*}}$ and Singh $\mathbf{M}^{2}$}

${ }^{1}$ Associate Professor of Medicine and Surgery, Baystate Medical Center, Tufts University School of Medicine, MA, USA

${ }^{2}$ Cardiology Fellow, Tulane University Health and Vascular, New Orleans, LA, USA

"Corresponding author: McGee WT, Associate Professor of Medicine and Surgery, Baystate Medical Center, Tufts University School of Medicine, Springfield, MA, USA, Tel: +413 794 5439; E-mail: William.mcgee@baystatehealth.org

Received date: July 21, 2016; Accepted date: September 03, 2016; Published date: September 07, 2016

Copyright:@ 2016 McGee WT, et al. This is an open-access article distributed under the terms of the Creative Commons Attribution License, which permits unrestricted use, distribution, and reproduction in any medium, provided the original author and source are credited.

\section{Case Report}

A 40-year-old man with no significant medical history presented with complaints of three weeks of progressively worsening fatigue, myalgia's and an episode of hematemesis. Two weeks prior to admission, he developed a dry cough that was associated with pleuritic chest pain. He denied chills or rigors and was unsure about fever. $\mathrm{He}$ also reported a 20-25 pounds weight loss with decreased appetite. He had an episode of hematemesis while coughing. He was born and raised in India and moved to the USA 15 years earlier. He took no medications. He had a history of prior alcohol use but stated he was sober. He denied drug use and had quit smoking a few years ago. He was employed at a gas station. He was previously in good health and able to do heavy physical labor without difficulty.

In the ER, he was hypoxic, tachycardia, and tachypneic with accessory muscle use. Examination of the chest revealed diffuse rhonchi. Additionally, he had palpable hepatomegaly and 1+ pitting pedal edema bilaterally. His dentition was poor and halitosis was noted. The chest X-ray showed a diffuse, symmetrical infiltration (Figure 1). An X-ray taken a year ago was normal.

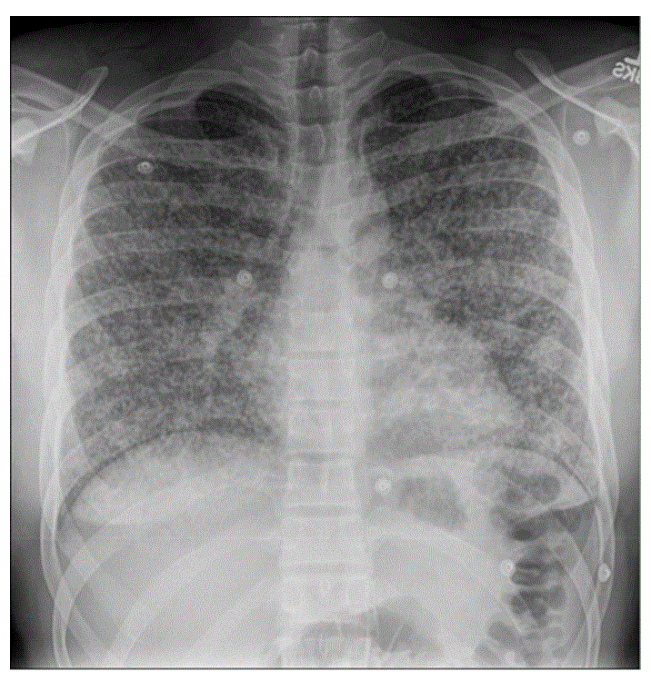

Figure 1: The chest X-ray showing a diffuse, symmetrical infiltration.

An arterial blood gas on $100 \%$ oxygen via a non-rebreather mask was $7.38 / 33 / 65 / 19$. The A-a gradient was 600 with a p/f ratio of 65 . He continued to worsen and was intubated and started on Ceftriaxone and
Azithromycin, INH and Rifampin after blood and sputum cultures were obtained. His ventilation setting were $100 \% \mathrm{FiO}_{2}$, a positive end expiratory pressure [PEEP] of $12 \mathrm{~cm} \mathrm{H}_{2} \mathrm{O}$ and a rate of $18 / \mathrm{min}$ with a tidal volume of $450 \mathrm{cc}$. Labs showed a white blood cell of 10.9, 78\% neutrophils, hematocrit of 24.5 , sodium of 118 , chloride 88 , bicarbonate of 19, alkaline phosphatase 218, ALT 61, total bilirubin 3.9, INR of 1.4 and a lactate of 3.7. Following intubation, he remained in refractory shock and was started on norepinephrine.

HIV, DFA, PCP, urine legionella antigen and blood and fungal cultures were negative. Bronchoscopy showed 1+ acid fast bacilli for tuberculosis; ethambutol and pyrazinamide were added. Shock improved and he was extubated after four days. A DNA probe of serum for Mycobacterium tuberculosis was positive. The sputum culture was positive for $M$. tuberculosis, non-resistant strain.

\section{Case Discussion}

This immunocompetent patient developed septic shock from a pulmonary tubercular infection which necessitated vasopressor and ventilatory support. As he had been living in a non-endemic region for many years, this was most likely a reactivation of a primary Ghon focus. Continued alcohol abuse is an important consideration regarding immunosuppression and susceptibility to reactivation. Reactivation disease typically follows a more benign course. His X-ray with the typical reticulo-nodular pattern although highly suggestive of miliary tuberculosis could also suggest multiple other infectious or non-infectious etiologies. The $\mathrm{PaO}_{2}: \mathrm{FiO}_{2}$ ratio was diagnostic of ARDS. For such patients the role of systemic steroids in the acute setting is unclear and therefore they were not started. Tuberculosis was suspected by the resident caring for the patient who had attended medical school in India. The attending physician had not previously initiated empiric anti-tuberculosis therapy at ICU admission over a 20 year career.

Septic shock from military tuberculosis has been described in immune-compromised patients. Septic ARDS at hospital presentation has been seen rarely in western populations and isolated case reports exist of such patients [1-3]. The largest case series of miliary tuberculosis related ARDS was a series of nine patients. Eight of the nine patients died and in two patients the diagnosis was made postmortem. [3]. The largest case series for ARDS secondary to miliary tuberculosis in the developing world is a series of six patients in India seen over a period of 12 years. All of these patients were HIV negative and two of these patients died as a result of the disease [4].

ARDS is a relatively rare manifestation of miliary tuberculosis. In a review of 109 patients in South Africa who had a diagnosis of miliary tuberculosis ARDS was present in only 7 patients [2]. Respiratory 
failure in miliary tuberculosis was described as early as 1969 by Godfine et al. [5]. Multiple case reports exist of an entity called "sepsis tuberculosis gravissima", an acute septic shock with ARDS and multiorgan failure from MTB although none after "date of last article". Altered mental status, hematological abnormalities, renal failure and death are common [6].

\section{Pathogenesis of ARDS in tuberculosis}

The development of ARDS in disseminated tuberculosis is poorly understood. In military tuberculosis the initial inflammatory reaction in the alveoli gives way to alveolar exudative deposits (containing polymorphonuclear leukocytes, erythrocytes and fibrin) and eventually caseating granulomas. This eventually leads to the signs and symptoms of ARDS but there is no general agreement on how this process is initiated and how it progresses. The role of a lipoprotein, Lipoarabinomannan, present in the mycobacterial cell wall causing activation of macrophages and resultant TNF- $\alpha$ and interleukin release causing damage to alveoli has also been postulated [7-8]. Few cases of ARDS in military tuberculosis have been reported in patients started on antibiotic therapy which suggests that the cell lytic process may be an initiating factor [8].

\section{Clinical Features}

Signs and symptoms of ARDS at initial presentation are rare and most patients present with sub-acute or chronic symptoms. History of constitutional symptoms may be present but it is often unhelpful as it fails to distinguish miliary TB from a viral illness. A high index of suspicion is needed for vulnerable patients. Migration from an endemic area to the USA is important as this case illustrates.

\section{Management}

ARDS in miliary tuberculosis is managed per established guidelines with the exception that patients would need early initiation of antitubercular therapy. In most cases the diagnosis of miliary tuberculosis is delayed and confirmatory tests can take days or longer. Mortality remains high in ARDS secondary to miliary tuberculosis, $33-85 \%$ in the limited in data available, especially because the causative etiology may not be clinically apparent [9]. Patients with disseminated intravascular coagulation, septic shock and pancytopenia have a poorer prognosis [9]. Pancytopenia is more commonly seen in immune-compromised patients.

$\mathrm{X}$-rays may show a reticulo-nodular pattern but this is often nondiagnostic as this pattern can be seen in other diseases such as pneumonitis, interstitial lung disease, pulmonary edema and sarcoidosis. High resolution CT scan can show diffuse nodular densities but this may be seen in other pneumonitis too. Bronchoalveolar lavage and in some cases other tissue diagnosis such as bone marrow and liver biopsies may be necessary to establish the diagnosis.
In case reports, the use of steroids has shown to help reduce the systemic toxicity and the exudative pulmonary damage [10-11].

\section{Conclusion}

Given the high rate of mortality of miliary tuberculosis early diagnosis and prompt management is essential. ARDS although uncommon, can occur in miliary tuberculosis and its management takes precedence as the histopathological diagnosis of tuberculosis can take days. Although anti-tubercular medications are not recommended in the management of ARDS per se, the possibility of miliary tuberculosis being the precipitating agent for ARDS should always be included in the differential diagnosis, especially in vulnerable populations. The importance of the experience of a multi-cultural workforce aided our patient directly.

\section{References}

1. Murray HW, Tuazon CU, Kirmani N, Sheagren JN (1978) The adult respiratory distress syndrome associated with miliary tuberculosis. Chest 73: $37-43$.

2. Maartens G, Willcox PA, Benatar SR (1990) Miliary tuberculosis: rapid diagnosis, hematologic abnormalities, and outcome in 109 treated adults. Am J Med 89: 291-296.

3. Piqueras AR, Marruecos L, Artigas A, Rodriguez C (1987) Miliary tuberculosis and adult respiratory distress syndrome. Intensive Care Med 13: $175-182$.

4. Mohan A, Sharma SK, Pande JN (1995) Acute respiratory distress syndrome (ARDS) in miliary tuberculosis: a twelve year experience. Indian J Chest Dis Allied Sci 38:157-162.

5. Godfine ID, Schachter H, Barclay WR, Kingdon HS (1969) Consumption coagulopathy in military tuberculosis. Ann Internal Med 71: 775-777.

6. Ahuja, Seema S, Ahuja SK, Phelps KR, Thelmo W, et al. (1992) Hemodynamic confirmation of septic shock in disseminated tuberculosis. Crit Care Med 20: 901-903.

7. Penner C, Roberts D, Kunimoto D, Manfreda J, Long R (1995) Tuberculosis as a primary cause of respiratory failure requiring mechanical ventilation. Am J Respir Crit Care Med 151: 867-872.

8. Zhang Y, Doerfler M, Lee TC, Guillenm B, Rom WN (1993) Mechanism of stimulation of interleukin and $1 \beta$ and TNF- $\alpha$ by Mycobacterium tuberculosis components. J Clin Invest 91: 2076-2083.

9. Roger PM, Deloffre P, Tiger F, Bernardin G, Corcelle P, et al. (1995) Prognosis of adult respiratory distress syndrome in tuberculosis patients: 4 Case reports. Presse Méd 24: 1021-1024.

10. Tokunaga N, Ichikawa Y, Kuboshiro M, Higashi T, Fujino K, et al. (1990) A case of miliary tuberculosis associated with acute respiratory failure during corticosteroid treatment of rheumatoid arthritis. Kekkaku 65: 539.

11. Marsepoil T, Griguere P, Mahassini B, Levesque P, Blin F (1990) Acute respiratory distress syndrome caused by tuberculosis. Ann Fr Anesth Reanim 9: 309-311. 